Published in "Physical Review E 88(1): 012818, 2013"

which should be cited to refer to this work.

\title{
Spreading in online social networks: The role of social reinforcement
}

\author{
Muhua Zheng (郑木华), ${ }^{1}$ Linyuan Lü (吕琳媛), ${ }^{2,3, *}$ and Ming Zhao (赵明) ${ }^{1, \dagger}$ \\ ${ }^{1}$ College of Physics and Technology, Guangxi Normal University, Guilin 541004, People's Republic of China \\ ${ }^{2}$ Institute of Information Economy, Alibaba Business School, Hangzhou Normal University, Hangzhou 310036, People's Republic of China \\ ${ }^{3}$ Department of Physics, University of Fribourg, Chemin du Musée 3, Fribourg CH-1700, Switzerland
}

\begin{abstract}
Some epidemic spreading models are usually applied to analyze the propagation of opinions or news. However, the dynamics of epidemic spreading and information or behavior spreading are essentially different in many aspects. Centola's experiments [Science 329, 1194 (2010)] on behavior spreading in online social networks showed that the spreading is faster and broader in regular networks than in random networks. This result contradicts with the former understanding that random networks are preferable for spreading than regular networks. To describe the spreading in online social networks, a unknown-known-approved-exhausted four-status model was proposed, which emphasizes the effect of social reinforcement and assumes that the redundant signals can improve the probability of approval (i.e., the spreading rate). Performing the model on regular and random networks, it is found that our model can well explain the results of Centola's experiments on behavior spreading and some former studies on information spreading in different parameter space. The effects of average degree and network size on behavior spreading process are further analyzed. The results again show the importance of social reinforcement and are accordant with Centola's anticipation that increasing the network size or decreasing the average degree will enlarge the difference of the density of final approved nodes between regular and random networks. Our work complements the former studies on spreading dynamics, especially the spreading in online social networks where the information usually requires individuals' confirmations before being transmitted to others.
\end{abstract}

\section{INTRODUCTION}

Diseases propagation, transmission of cultures and information, or behaviors spreading are common phenomena in human society and have attracted much attention for a long time [1-3]. Although these phenomena possess some similar properties, it is still difficult to analyze them under a unified framework or use analogous models. In general, all these processes can be classified into two classes: epidemic spreading and information spreading. Their differences are mainly embodied in the following three aspects: (i) Role of spreader. People are passive to be affected in epidemic spreading while active to make decisions in information spreading, like approving or disapproving some news. The social status or influences and personal interests of spreaders may play important roles in information spreading while count for little in epidemic contagion. (ii) Property of disseminule (disseminule is a general term for information, rumor, behavior and so on). The information has timeliness and reliability requirements while diseases can be active for a long time and are invasive during the spreading process. For example, influenza virus can exist for thousands of years in human society, but rumor may have only several days life length [4]. (iii) Spreading path. The spreading of diseases from person to person usually requires physical contacts while the information propagation is mostly via online connections presently besides the traditional face-to-face communications.

Due to the above differences, the spreading dynamics of disease and information should be described by different models. Up to now, two classical models, namely susceptible-infected-

\footnotetext{
*linyuan.lv@gmail.com

†zhaom17@gmail.com
}

susceptible (SIS) model and susceptible-infected-recovered (SIR) model, are proposed to describe disease propagation [1,2,5-9]. These two models found further effectiveness in their explanation of traditional information spreading, where individuals communicate face to face (to some extent like physical contacts in epidemic spreading) and are easier to be convinced (refers to higher probability of approval) [10-14]. However, it has been pointed out that the SIS and SIR models fail to explain the information or behavior spreading in online social networks [15-17]. Since only a few connections are the copies of the off-line relationships, the connections in online social networks are usually weaker than the face-to-face communications. It is common that the information from our best friends (i.e., strong ties) has higher probability to be approved than those from just acquaintances (i.e., weak ties). Therefore, the information spreading in online social networks should be much different from the epidemic spreading or the off-line information spreading at the microscopic or macroscopic level. To date, some models that describe information spreading process have been presented $[18,19]$, but there is still not a clear picture on this issue. In 2010, Centola conducted an experiment of behavior spreading in online social networks; although behavior and information are different disseminules, we believe they share some similar properties and the behavior spreading process will make use of the information spreading. For example, individuals often make decisions before they transmit the information or action signal, and individuals take action only after they received the action information from the neighbors. So, in this paper, we modeled Centola's online experiment and hoped that our research on behavior spreading can be used to describe information spreading. Since information spreading is a part in behavior spreading process, to avoid confusion, only the concept of information spreading is used in the discussion of 
information itself and the behavior spreading in the following narration.

In Centola's experiment, it is observed that social reinforcement played a crucial role in the online spreading of behavior [20]. The so-called social reinforcement is defined as the situation in which an individual requires multiple prompts from neighbors before adopting an opinion or behavior [21-27]. The experiment showed that a single signal has a very weak effect on individuals' decision-making and that when individuals receive multiple signals they are more likely to accept the information and adopt the behavior. As a result, the behavior spreads faster and farther in high-clustered regular networks than in random ones, and the reason is that in this case the clustering coefficient is large in regular networks, which makes individuals receive redundant signals easier. This result contradicts with the former understanding that random networks are preferable for information spreading [11,28].

In this paper, we take into account the main difference between epidemic spreading and information spreading and propose a model that emphasizes the effect of social reinforcement on spreading process. Centola's experiment shows the behavior spreading macroscopically, and our model is proposed in microscopic level; however, simulation results on modeled networks show that our model can well describe the spreading behavior, especially in online social networks. Specifically, the spreading results are mainly affected by two key factors, the primary spreading rate (the probability of an individual adopts the behavior after receiving its information at the first time) and the strength of social reinforcement. Performing our model on regular and random networks, four regions are found according to the spreading range: (i) Regular networks winning region. This region corresponds to a small primary spreading rate and a considerable strength of social reinforcement, where the spreading is faster and farther in regular networks than in random ones. This result is consistent with the online experiment on behavior spreading [20]. (ii) Random networks winning region. This region corresponds to a medium primary spreading rate and weak social reinforcement effect, which is consistent with the results from former studies on off-line information spreading by using epidemic spreading models $[11,28]$. (iii) Absorbing region. In this region, the primary spreading rate is too small and the strength of social reinforcement is too weak; thus, the information cannot spread out. (iv) Over active region. In this region, the primary spreading rate is too large and no matter how strong (or weak) the social reinforcement strength is, the information will spread to the whole networks and there is no much difference between the spreadings on regular and random networks. In addition, our model can degenerate to the SIR model at certain parameters. The effects of average degree and network size on the spreading process are also analyzed and the results reaffirm the importance of the social reinforcement in the behavior spreading process and prove the anticipation of Centola's experiments. Our work complements the former studies on spreading dynamics, especially the spreading in online social networks.

The rest of this paper is organized as follows: In Sec. II, Centola's experiment is analyzed in depth and a mathematical model to describe this experiment is proposed. In Sec. III, the regular and random network models used in this work are introduced. And then, the simulation results of our model are presented. The effects of social reinforcement, average degree, and network size on spreading results are discussed later. In Sec. IV, the discussion and conclusion are presented.

\section{MODEL ON THE BEHAVIOR SPREADING IN ONLINE SOCIAL NETWORKS}

In 2010, Centola studied the effects of network structure on the spreading of health behavior in online social networks [20,29]. In his experiment, 1528 participants were recruited and were randomly embedded in six networks that represented Internet-based health communities. In the six networks, three are regular lattices and the rest are random ones with the same size and average degree as the corresponding regular lattices. In the experiment, the participants could not contact others directly but could receive emails informing them their neighbors' activities. Once a participant received an email, she would make a decision whether to adopt the behavior or not, and if she decided to take the same action, emails would be sent to her neighbors who had not adopted the behavior to inform them her activity. By and by, the behavior would spread out in the network. From the experiment, the author found that for the adoption of behaviors, social reinforcement is a crucial factor for the spreading process, namely individuals are more likely to adopt the behavior when receiving more signals, leading to the result that the behavior spreads faster and farther in regular networks than in random ones.

After carefully studying this experiment, we find that besides the social reinforcement, the primary spreading rate, denoted by $\lambda_{1}$, is another key factor for behavior spreading in online social networks, and only when $\lambda_{1}$ takes small value will the social reinforcement take effect on the spreading process. A large primary spreading rate indicates that when an individual received the information about her neighbor's activity at the first time, she has higher probability to accept and take the same action. In this situation, the factor of social reinforcement is meaningless. In the following part, according to the experiment and our analysis, a spreading model will be proposed and its validity will be discussed.

First of all, we describe the spreading process in the model. At the beginning, there emerges an individual who knows some information, and she will send signals to all her neighbors. All her neighbors receive the signal but only a part of the neighbors will believe the information; they are called approved individuals. Then the approved individuals will send their states to their own neighbors. By and by, the information will spread out. During this process, the more times the individuals receive the signal, the easier it is for them to approve the information. Thus, there are four states in the system for individuals [18]: (i) Unknown state. The individual has not received the signal, analogous to the susceptible state of the SIR model. (ii) Known state. The individual is aware of the information (i.e., received the signal at least once) but does not approve. (iii) Approved state. The individual approves the information and then sends the signal to all her neighbors. (iv) Exhausted state. After transmitting the approved signal, the individual will never transmit it again, analogous to the recovered state in the SIR model. 
In our model, at the beginning, one node is randomly chosen as the "seed" and all others are in the unknown state. This seed node knows some information and transmits the signal to all her neighbors and then becomes exhausted. At each time step, if an individual (in either an unknown or a known state) receives the signal, she will change to approved state with probability $\lambda_{m}$, where $m$ is the number of times the individual has received the signal, and larger $m$ corresponds to larger $\lambda_{m}$. Thus, the more times the individual receives the signal, the easier it is for her to approve the information. Once the individual approves the information, she will transmit it to all her neighbors in the next time step and then becomes exhausted. If an individual, in either an unknown or a known state, does not receive any signal in the current time step, nothing will happen no matter how many times this individual has received the signal. In our model, the updates of the states of the individuals are synchronous, i.e., all the individuals evaluate their states simultaneously. And when no new individuals change their states any more, the spreading process goes to the end. It is worth noting that each connection is used at most once in our model. This is a significant difference between epidemic spreading and information spreading, where individuals always run the risk of being infected by their infectious neighbors, while people rarely transmit the same news to one person once and once again.

Now the key point is how to define $\lambda_{m}$. When an individual receives the signal at the first time, she will approve the information with probability $\lambda_{1}$, which is the so-called primary spreading rate. And when an individual receives the signal twice, she will approve the information with probability $\lambda_{2}$, and when she receives the signal three times the probability will be $\lambda_{3}$, and so on. In our model, the approving probability with different times that an individual receives the signal is defined as the following:

$$
\begin{aligned}
\lambda_{1} & =\lambda_{1}, \\
\lambda_{2} & =\lambda_{1}+b \times\left(1-\lambda_{1}\right), \\
\lambda_{3} & =\lambda_{2}+b \times\left(1-\lambda_{2}\right), \\
& \vdots \\
\lambda_{m} & =\lambda_{m-1}+b \times\left(1-\lambda_{m-1}\right),
\end{aligned}
$$

where $b \in[0,1]$ is the social reinforcement strength. Larger $b$ means redundant information will have stronger influence on the individuals. The iterative Eq. (1) indicates that if the individual has received the signal $m$ times, the approving probability will increase $b\left(1-\lambda_{m-1}\right)$ compared with $\lambda_{m-1}$ (i.e., receiving the signal $m-1$ times). The increase can be considered as an increment of spreading rate converted from disapproving probability $1-\lambda_{m-1}$ under the effect of social reinforcement. There are two extreme cases: When $b=0$, it means no social reinforcement effect, and this model degenerates to the standard SIR model; When $b=1$, the social reinforcement effect is very strong, and the probability of approval for $m \geqslant 2$ equals 1 , meaning that once an individual receives the signal twice, she will definitely approve the information. In summary, the above Eq. (1) can be simplified
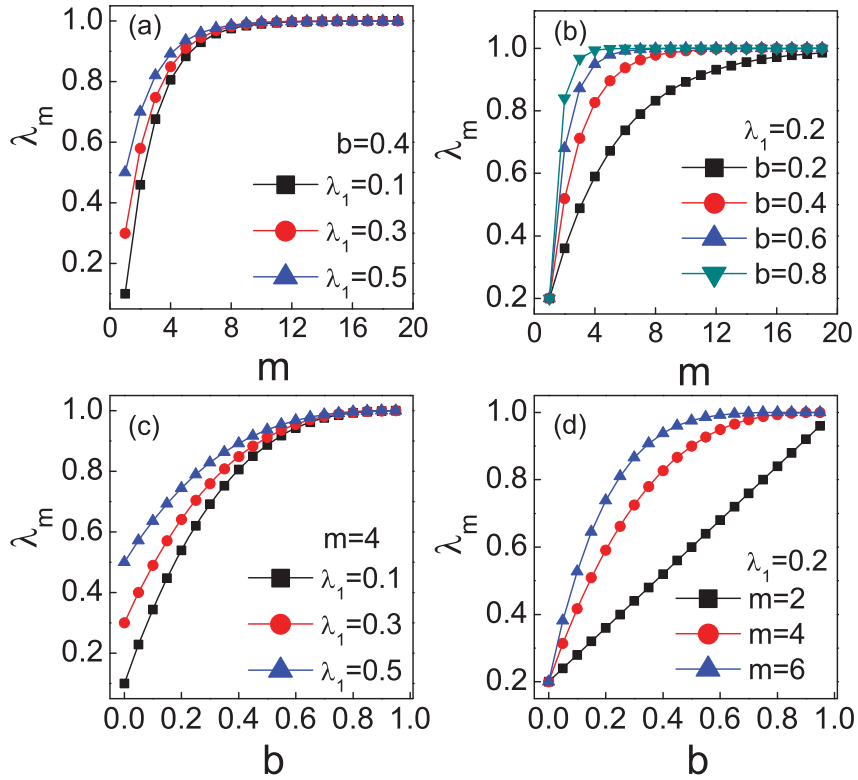

FIG. 1. (Color online) The spreading rate $\lambda_{m}$ as a function of $m$ at different (a) primary spreading rate $\lambda_{1}$ and (b) social reinforcement strength $b . \lambda_{m}$ as a function of $b$ at (c) different $\lambda_{1}$ and (d) different $m$.

as the following:

$$
\lambda_{m}=\left\{\begin{array}{cc}
\lambda_{1} & b=1, \quad m=1 \\
1 & b=1, \quad m \geqslant 2 ; \\
1-\left(1-\lambda_{1}\right)(1-b)^{m-1} & 0 \leqslant b<1, \quad m \geqslant 1 .
\end{array}\right.
$$

In Eq. (2), when parameters $\lambda_{1}$ and $b$ are kept fixed, $\lambda_{m}$ will increase monotonically with the increasing of $m$, i.e., the more times the individuals receive the signals, the larger the spreading rate will be. And $b$ has the same effect on $\lambda_{m}$. The change of $\lambda_{m}$ with parameters $m$ and $b$ are shown in Fig. 1.

\section{SIMULATION RESULTS}

\section{A. Experiment networks}

We perform our model on two regular networks, namely Hexagonal lattice and Moore lattice [29], and their corresponding homogeneous random networks. The sketch maps of the modeled networks are shown in Fig. 2. Both the Hexagonal and Moore lattice networks with size $N=H \times L$ will be

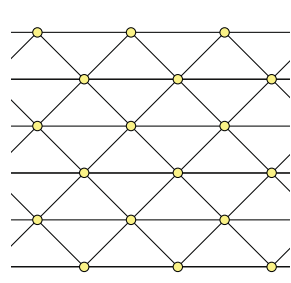

(a)

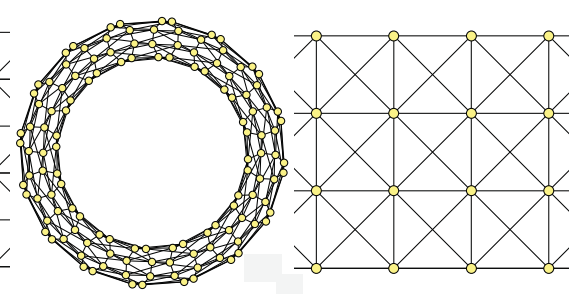

(b) (c)
FIG. 2. (Color online) (a) The neighborhood structure for the Hexagonal lattice network and (b) the corresponding network in periodic boundary condition. (c) The neighborhood structure for the Moore lattice network. 
constructed in periodic boundary condition, or in simple term, they are located on tori (i.e., toroidal surfaces). In all simulations, the ratio of the number of nodes in the row and column is fixed at 2:1, as in Centola's experiment. We have checked the effects of the ratio on our experiment and found that in a broad range of the ratio the results have no essential difference. The homogeneous random networks are constructed by using the Maslov-Sneppen small world rewiring technique [30,31], which is described as the following: At each time step, a pair of edges A-B and C-D are randomly selected, and these two edges are rewired to be A-D and B-C. During the rewiring process, self-connection and reconnection are forbidden. In order to obtain a completely random topology, this operation should be repeated many times. In our work, we implement $p N_{E}$ steps, where $p$ indicates the randomness of the network and $N_{E}$ is the number of connections in the network. Theoretically speaking, a homogeneous random network is obtained only for $p \rightarrow \infty$. When $p>1$, the simulation shows that the topological statistics are very close to those of random networks. So, we here set $p=10$.

\section{B. Effects of social reinforcement}

In our simulation, $\rho_{t}$ denotes the density of approved nodes in time step $t$, and $\rho_{\infty}$,reg and $\rho_{\infty}$,ran denote the density of approved nodes at the final steady states in regular networks and random networks, respectively. To compare the spreading range between these two types of networks at the final steady states, the difference $\delta_{\rho}=\rho_{\infty}$,reg $-\rho_{\infty}$,ran are calculated, and positive value of $\delta_{\rho}$ indicates the spreading range is broader in regular networks than in random networks, and negative value indicates the reverse case.

Figures 3(a) and 3(b) plot $\rho_{\infty}$,reg and $\rho_{\infty}$,ran in the parameter space $\left(\lambda_{1}, b\right)$ in Hexagonal lattice and the corresponding homogeneous random networks, respectively. By comparison, it is clearly seen that in the space where $b$ is larger but $\lambda_{1}$ is small, the spreading range is much larger in regular networks than in random networks. However, in the space where $\lambda_{1}$ is a little larger (at about 0.3) and $b$ is small, the spreading range is larger in random networks than in regular ones. Figure 3(c) plots the difference of $\rho_{\infty}$ between the Hexagonal lattice and its corresponding homogeneous random networks in the space $\left(\lambda_{1}, b\right)$. There are two islands in the parameter space: One is region I with small $\lambda_{1}$ and large $b$, where the spreading range is broader in regular networks, consistent with Centola's experiment [20]; the other one is region II, with relatively larger $\lambda_{1}$ and smaller $b$, where the spreading range is broader in random networks, consistent with the former results that spreading in random networks is farther than in regular networks $[11,28]$. Except the two islands, the value of $\delta_{\rho}$ in all the other regions is about 0 . However, region III (with small $\lambda_{1}$ and small $b$ ) and region IV (with larger $\lambda_{1}$ ) present totally different situations: In region III, the primary spreading rate is too small and the strength of social reinforcement is too weak, thus, the information cannot spread out in both kinds of networks, and as a result, the difference of spreading range is about 0 ; in region $\mathrm{IV}$, the primary spreading rate is too large and no matter how strong (or weak) the social reinforcement strength is, the information will spread to almost the whole networks and there is not much difference between
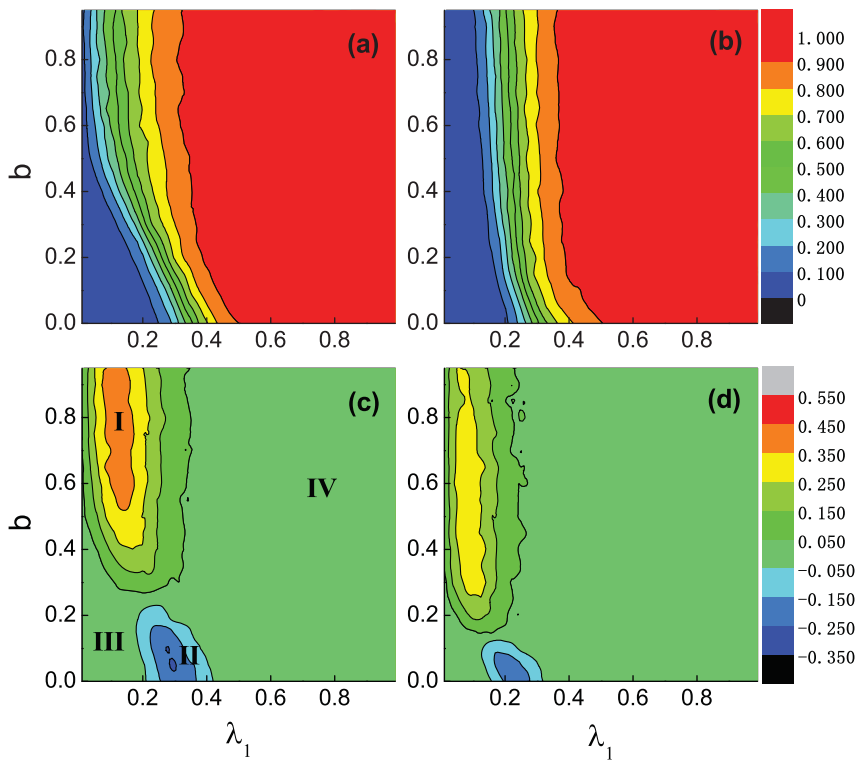

FIG. 3. (Color online) (a) $\rho_{\infty}$,reg in Hexagonal lattice, (b) $\rho_{\infty, \text { ran }}$ in the corresponding homogeneous random networks of Hexagonal lattice, and (c) the difference $\delta_{\rho}$ between the Hexagonal lattice and its corresponding homogeneous random networks in the parameter space $\left(\lambda_{1}, b\right)$. (d) The difference $\delta_{\rho}$ between the Moore lattice and its corresponding homogeneous random networks. The results are obtained by averaging over 10000 times of implements ( 100 network configurations and 100 initial states of each configuration).

the spreadings on regular and random networks; thus, the difference of spreading range is approximately 0 .

The result of $\delta_{\rho}$ between Moore lattice and its corresponding homogeneous random networks is also shown in Fig. 3(d). Interestingly, region $\mathrm{I}$ in this case covers a larger range of $b$ compared with Fig. 3(c), indicating that a larger average degree may decrease the threshold of $b$ and make regular networks defeat random networks, even when $b=0.145$. The reason for this phenomenon is that it is easier for individuals to receive signals from neighbors in networks with $k=8$ than $k=6$. For the case of different system size, the situation of larger $N$ can be considered in our model, which is hard to realize in online experiments. The simulation results show the locations of regions I and II are almost the same for different system size, and only the value of the difference $\delta_{\rho}$ becomes more prominent.

To further show the interpretability of our model to Centola's experimental results, in Fig. 4 we plot the number of approved nodes as a function of time on regular networks (black squares) and on random networks (red circles). For comparison, we copy the result from Centola's experiment in Fig. 4(a), where the solid black circle and open triangle curve are time series of the fraction of individuals who adopted the behavior in clustered Hexagonal lattice network and homogeneous random network with the same degree, respectively. From the figure it can be seen that for both the spreading speed at the beginning and the spreading range at the final steady state, regular network won a victory over the random one. Figure 4(b) gives the result with parameters $\lambda_{1}=0.18$ and $b=0.40$. The curves show almost the same trend as the experiment: The spreading is faster and farther 

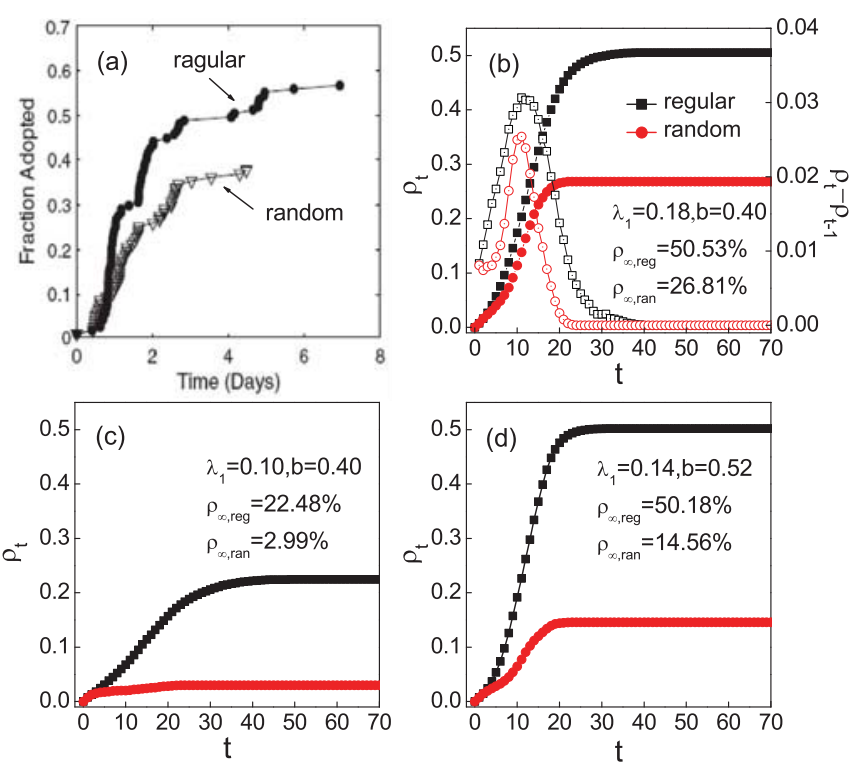

FIG. 4. (Color online) (a) The adoption proportion of a health behavior spreading through clustered-lattice (solid black circles) and random (open triangles) social networks (which is cited from the article [20]). (b-d) The density of approved nodes as a function of time in regular networks (black squares) and in homogeneous random networks (red circles). In (b), the open dot curves indicate the increment of density of approved nodes at each time step $\rho_{t}-\rho_{t-1}$. The network size $N=16 \times 8=128$ and degree $k=6$. The results are obtained by averaging over 10000 implements.

in regular networks than in random ones. To quantitatively describe the spreading speed, we plot the increment value of approved nodes density at each time step, namely $\rho_{t}-\rho_{t-1}$, in regular networks (open black square) and random networks (open red circle). The trends of the two curves are almost the same. At the beginning, the spreading speed in both networks increase sharply, and then the two curves reach their own maximum almost at the same time, which denotes the occurrence of outbreak, and after that the curves decrease sharply with the increasing of $t$. During the whole process the value of $\rho_{t}-\rho_{t-1}$ in regular networks is always larger than in random networks, which indicates the spreading speed in regular networks is faster. $\rho_{t}-\rho_{t-1}=0$ indicates the spreading has stopped and the spreading range has reached the maximum. From the above results, it can be concluded that our model is appropriate to describe the behavior spreading in online social networks.

In the parameter space, there are some special points presenting some interesting phenomena. Two typical examples are shown in Figs. 4(c) and 4(d). In Fig. 4(c), the parameter $\lambda_{1}=0.1$, which is too small to make the information spread out in random networks, while in regular networks, with a strong social reinforcement, namely $b=0.40$, there are still $20 \%$ individuals who approved the information. In Fig. 4(d), with the primary spreading rate $\lambda_{1}=0.14$ and the social reinforcement strength $b=0.52$, there is an extremely large difference of $\rho_{\infty}$ between regular and random networks. The above results show that by regulating the primary spreading rate and social reinforcement strength, the spreading in regular networks and in random networks show diverse
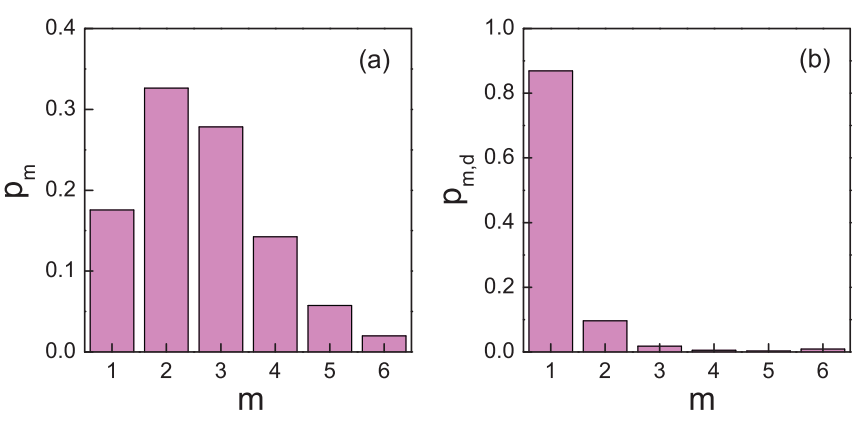

FIG. 5. (Color online) The distribution of (a) approved nodes $p_{m}$ and (b) disapproved nodes $p_{m, d}$ after receiving the signal $m$ times. The parameters are $\lambda_{1}=0.18, b=0.4$, and $k=6$. The data are obtained from 10000 samples with fixed network size $N=16 \times 8=128$. The statistics are obtained at the steady state.

properties. It has been found that our model can describe Centola's experiment rather well with $\lambda_{1}=0.18$ and $b=$ 0.40 , thus, hereinafter, we will concentrate our study on this case.

We have known that the social reinforcement is crucial for the behavior spreading, and then a natural question emerges: How strong does social reinforcement affect individuals? In other words, how many times do individuals have to hear the information before approving it? To answer this question, we define $p_{m}$ as the ratio of the number of approved individuals after receiving the signals $m$ times to the number of final approved individuals. The distribution of $p_{m}$ at the steady state is shown in Fig. 5(a). It can be seen that only $17.6 \%$ of the total approved individuals approve the information after receiving the signal once (i.e., $m=1$ ). When $m=2, p_{m}$ increases to more than $30 \%$, and after that with the increasing of $m, p_{m}$ decreases, but the values of $p_{3}$ and $p_{4}$ are still very large. Similar to $p_{m}$, we further define $p_{m, d}$ as presenting the probability of individuals who have received the signal $m$ times but do not approve it. From Fig. 5(b), we can see that more than $80 \%$ of individuals don't approve the signal after receiving only one signal, indicating that most individuals are affected by the social reinforcement effect. That is to say that the redundant signals can significantly increase the likelihood of approval. Therein, the effect of the second signal is the most significant. This conclusion has been tested by Centola's experiment [20].

\section{Effects of average degree and network size}

In our model, the average degree is increased by adding edges between each node and its next-nearest neighbors. And when changing the network size, the number of nodes in row and column keeps fixed ratio at 2:1. In this part, we focus on Hexagonal lattice. The effects of average degree $k$ on the density of approved individuals at the steady states $\rho_{\infty}$ for regular (filled dot solid line) and random (open dot dash line) networks at different network size are shown in Fig. 6(a). Clearly, $\rho_{\infty}$ increases monotonically with $k$ for both kind of networks, and with the same degree, the spreading in regular networks is generally broader than in random ones. Why regular networks perform better? It is mainly resulted from the ability of receiving redundant signals. When $k$ is 

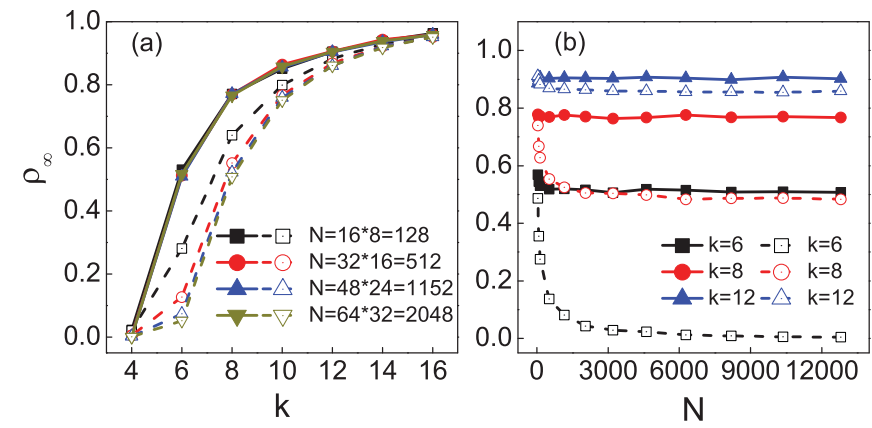

FIG. 6. (Color online) (a) The density of approved nodes $\rho_{\infty}$ on regular (filled dot solid line) and random (open dot dash line) networks against the degree $k$ given different $N$ values. (b) The density of approved nodes $\rho_{\infty}$ on regular (filled dot solid line) and random (open dot dash line) networks against $N$ given different degree $k$. The parameters are $\lambda_{1}=0.18, b=0.4$. The data are obtained from 10000 samples.

small, individuals have very few connections to others. In this case, a high clustering coefficient can help to obtain more signals in the low-nutrient situation. Therefore, with the same average degree, the regular network whose clustering coefficient is larger has advantage for spreading when the effect of social reinforcement is taken into account. However, when $k$ is large, in both random and regular networks, there are enough opportunities for individuals to obtain redundant signals via the sufficient connections to others. At this moment, the advantage of regular networks becomes negligible although owning larger clustering coefficient. For example, when $k=$ 16 in both networks, about $95 \%$ individuals will approve the information. For the special case when $k=4$, neither the clustering coefficient (about zero) nor the average degree of these two networks is large enough to make the individuals obtain enough redundant signals, so that the information in the two kinds of networks will not spread out, i.e., $\rho_{\infty}$ is almost zero.

Furthermore, in Fig. 6(b) we investigate the dependence of the number of final approved individuals on the network size given different average degree $k$. It shows that when $k$ is small, $\rho_{\infty}$ in random networks decreases with the increasing of network size (steep at the beginning and slow down later), while in regular networks $\rho_{\infty}$ keeps almost the same. The reason is in random networks, the clustering coefficient will decrease with the increase of the network size. As we have analyzed above, the networks with higher clustering coefficient have advantage of receiving more signals and, therefore, lead to larger spreading range. However, the clustering coefficient of regular networks in our experiment keeps constant; thus, the individuals will not be affected so much for receiving redundant signals, leading to a relatively stable spreading ranges. Again, when $k$ is large, the difference between random and regular networks is not prominent due to the higher probability to obtain redundant signals via large number of connections to others. Generally speaking, our conclusion is accordant with Centola's anticipation that when the network is sparse, increasing the network size or decreasing the average degree may enlarge the difference of $\rho_{\infty}$ between regular and random networks, and when the average degree is large enough, the influence of topological difference on the spreading ability is no longer notable [29].

\section{CONCLUSION AND DISCUSSION}

In summary, we propose a four-status model to describe the spreading in online social networks, namely an unknownknown-approved-exhausted states model. We carry out the simulations on Hexagonal lattice and Moore lattice networks with periodic boundary condition, which have similar topological structure with the networks in Centola's online experiments, and further compare the results with simulations on the corresponding random networks. The results show that the density of final approved nodes is strongly affected by the combined effects of the primary spreading rate $\lambda_{1}$ and the social reinforcement $b$, which are two key factors in the spreading process. In the parameter space $\left(\lambda_{1}, b\right)$, four regions (labeled in Fig. 3) are found according to the spreading range. It can be seen that even if the primary spreading rate $\lambda_{1}$ is too small to make the spreading breakout, with stronger social reinforcement strength $b$, regular networks can promote the diffusion better than random networks. In other words, under certain conditions, the spreading is faster and farther in regular networks than in the corresponding random networks (see region I in Fig. 3). These results support Centola's experiment [20]. The best parameters to explain the results of Centola's experiment are $\lambda_{1}=0.18$ and $b=0.4$. When $\lambda_{1}$ is a little larger and $b$ is small (corresponding to region II in Fig. 3), the spreading in regular networks is not as good as in random ones. This result is accordant with the traditional understanding of network spreading $[11,28]$. In the extreme case, when $\lambda_{1}$ is very large, the individuals have high probability to approve no matter how strong (or weak) the social reinforcement strength is. Therefore, the information will spread to almost the whole network, leading to not much difference between the spreadings on regular and random networks. When $b=0$, namely there is no social reinforcement effect, our model degenerates to the standard SIR model. We further study the effects of average degree and network size on spreading and the result is again accordant with Centola's anticipation that in region I increasing the network size or decreasing the average degree can enlarge the difference of the density of final approved nodes $\rho_{\infty}$ between regular and random networks.

Although our model is simple, it can very well explain Centola's experiment of behavior spreading in online social networks [20], and we believe that our model is easy to expand to describe other spreading processes, such as rumor or information that are usually required confirmations before being transmitted. Of course, some real experiments on these spreading processes are needed to further test the validity of the model. Besides, the study on the effects of other important structural features, such as clustering coefficient, average distance, etc., are lacking, which will be expected in future studies. Moreover, in our work we only consider the networks with homogenous degree, namely each node has the same number of neighbors, but in real world networks, the degrees generally distribute heterogeneously $[13,32,33]$. Thus, to study the spreading process on such networks and to find out the key spreaders are of great practical significance [34-36], which are also set as our future tasks. 


\section{ACKNOWLEDGMENTS}

We acknowledge Tao Zhou and Haifeng Zhang for valuable discussions. This work is supported by the $\mathrm{Na}$ tional Natural Science Foundation of China (Grant No.
11165003) and by the Program for Excellent Talents in Guangxi Higher Education Institutions. Linyuan Lü acknowledges the research start-up fund of Hangzhou Normal University.
[1] N. T. J. Bailey, The Mathematical Theory of Infectious Diseases and It Applications (Hafner Press, New York, 1975).

[2] R. M. May and R. M. Anderson, Infectious Diseases of Humans. Dynamics and Control (Oxford University Press, Oxford, 1992).

[3] A. Vespignani, Nature Phys. 8, 32 (2012).

[4] F. Wu and B. A. Huberman, Proc. Natl. Acad. Sci. USA 104, 17599 (2007).

[5] R. Pastor-Satorras and A. Vespignani, Phys. Rev. Lett. 86, 3200 (2001).

[6] V. M. Eguiluz and K. Klemm, Phys. Rev. Lett. 89, 108701 (2002).

[7] M. Boguna, R. Pastor-Satorras, and A. Vespignani, Phys. Rev. Lett. 90, 028701 (2003).

[8] R. M. May and A. L. Lloyd, Phys. Rev. E 64, 066112 (2001).

[9] M. E. J. Newman, Phys. Rev. E 66, 016128 (2002).

[10] A. Sudbury, J. Appl. Prob. 22, 443 (1985).

[11] D. H. Zanette, Phys. Rev. E 64, 050901(R) (2001).

[12] D. H. Zanette, Phys. Rev. E 65, 041908 (2002).

[13] Z. Liu, Y.-C. Lai, and N. Ye, Phys. Rev. E 67, 031911 (2003).

[14] Y. Moreno, M. Nekovee, and A. F. Pacheco, Phys. Rev. E 69 , 066130 (2004).

[15] C. Castellano, S. Fortunato, and V. Loreto, Rev. Mod. Phys. 81, 591 (2009).

[16] J. L. Iribarren and E. Moro, Phys. Rev. Lett. 103, 038702 (2009).

[17] J. L. Iribarren and E. Moro, Phys. Rev. E 84, 046116 (2011).

[18] L. Lü, D.-B. Chen, and T. Zhou, New J. Phys. 13, 123005 (2011).

[19] T. House, J. R. Soc. Interface. 8, 909 (2011).

[20] D. Centola, Science 329, 1194 (2010).
[21] S. N. Majumdar and P. L. Krapivsky, Phys. Rev. E 63, 045101(R) (2001).

[22] F. J. Perez-Reche, J. J. Ludlam, S. N. Taraskin, and C. A. Gilligan, Phys. Rev. Lett. 92, 218701 (2004).

[23] H. P. Young, Amer. Econ. Rev. 99, 1899 (2009).

[24] C. de Kerchove, G. Krings, R. Lambiotte, P. Van Dooren, and V. D. Blondel, Phys. Rev. E 79, 016114 (2009).

[25] B. Karrer and M. E. J. Newman, Phys. Rev. E 82, 016101 (2010).

[26] J.-P. Onnela and F. Reed-Tsochas, Proc. Natl. Acad. Sci. USA 107, 18375 (2010).

[27] F. J. Pérez-Reche, J. J. Ludlam, S. N. Taraskin, and C. A. Gilligan, Phys. Rev. Lett. 106, 218701 (2011).

[28] T. Zhou, Z.-Q. Fu, and B.-H. Wang, Prog. Nat. Sci. 16, 452 (2006).

[29] Materials and methods are available as supporting material on Science Online. www.sciencemag.org/cgi/content/full/ 329/5996/1194/DC1.

[30] S. Maslov and K. Sneppen, Science 296, 910 (2002).

[31] S. Maslov, K. Sneppen, and U. Alon, in Handbook of Graphs and Networks: From the Genome to the Internet, edited by S. Bornholdt and H. G. Schuster (Wiley-VCH, Berlin, 2003).

[32] A.-L. Barabási and R. Albert, Science 286, 509 (1999).

[33] R. Pastor-Satorras and A. Vespignani, Phys. Rev. E 63, 066117 (2001).

[34] M. Kitsak, L. K. Gallos, S. Havlin, F. Liljeros, L. Muchnik, H. E. Stanley, and H. A. Makse, Nature Phys. 6, 888 (2010).

[35] L. Lü, Y.-C. Zhang, C. H. Yeung, and T. Zhou, PLoS ONE 6, e21202 (2011).

[36] D.-B. Chen, L. Lü, M. S. Shang, Y.-C. Zhang, and T. Zhou, Physica A 391, 1777 (2012). 